\title{
Emoji and rapport management in Spanish WhatsApp chats
}

\section{Agnese Sampietro}

sampietr@uji.es

University Jaume I, Faculty of Humanities and Social Sciences, Department of Languages and European Cultures, Av. Sos Baynat S/n, 12071 Castellón de la Plana, Spain

*Tel.: +34964 729846 .

Abstract

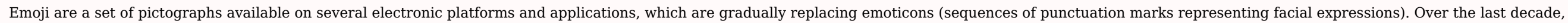

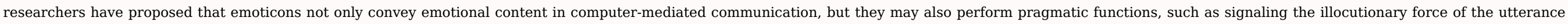

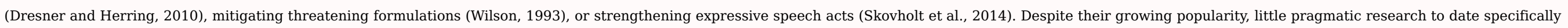

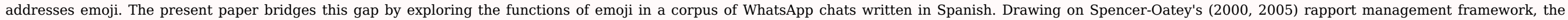

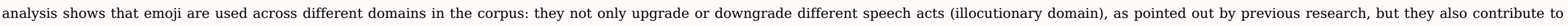

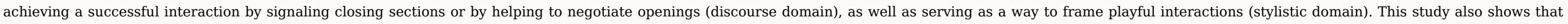
some practices related to the use of emoji may be influenced by Spanish culture.

Keywords: Emoji; Computer-mediated communication; WhatsApp; Rapport management; Politeness; Spanish

\section{Introduction}

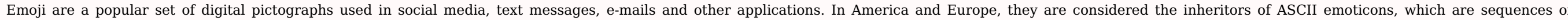

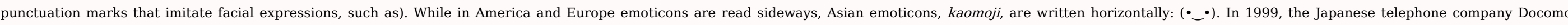

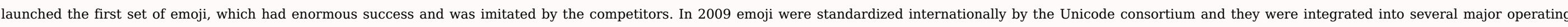

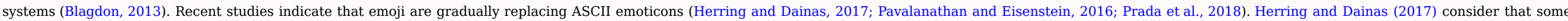
design features (emoji are small, static, simple but not too minimalistic) may have fostered their success.

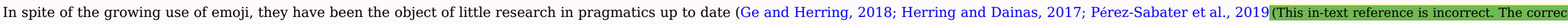

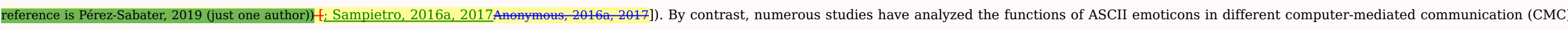

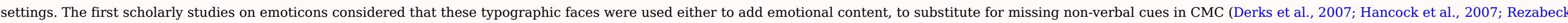

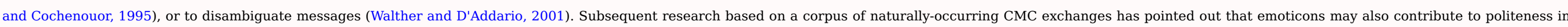
CMC (Dresner and Herring, 2010; Markman and Oshima, 2007; Skovholt et al., 2014; Vandergriff, 2013).

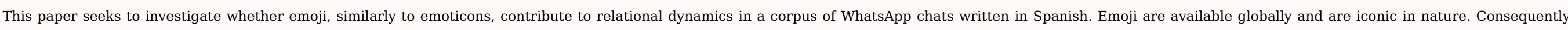

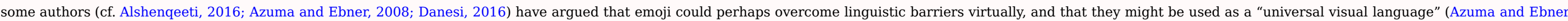

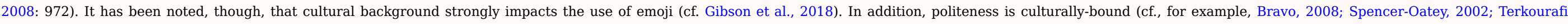

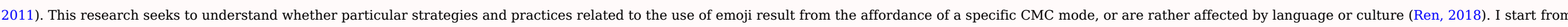

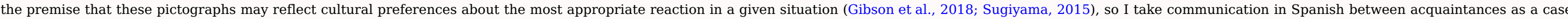

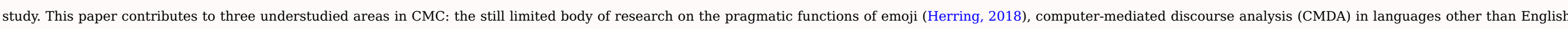

(Danet and Herring, 2003; Ren, 2018), and the study of everyday computer-mediated conversation (Georgakopoulou, 2011; Herring, 2018). 


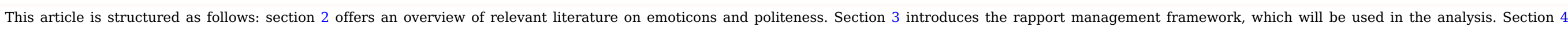
discusses the methodology (data, data collection, and procedure) and section 5 reviews the analysis of the corpus. Finally, the discussion and conclusions are presented (section 6).

\section{Emoticons and emoji: beyond affect}

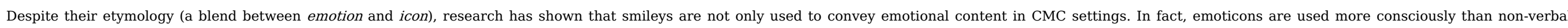

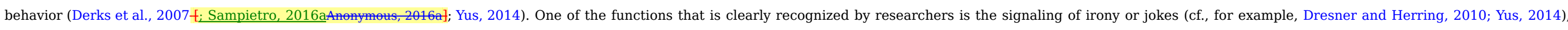

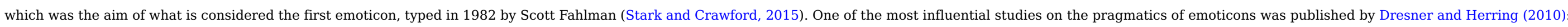

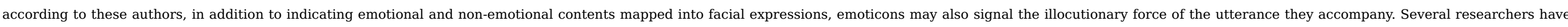

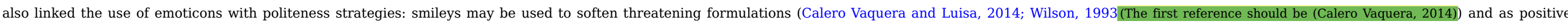

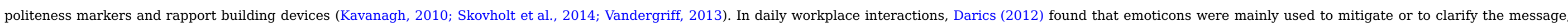
frequently with the purpose of reaching a successful cooperation.

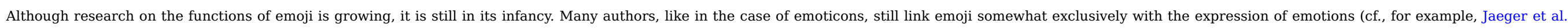

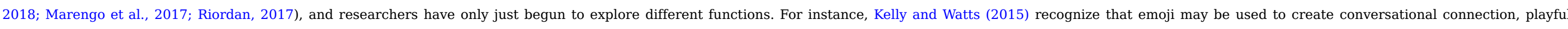

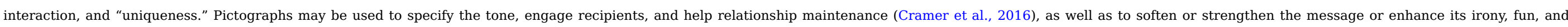

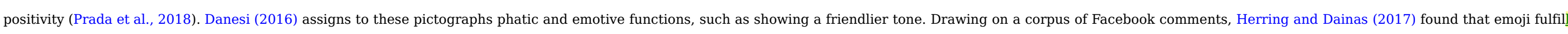

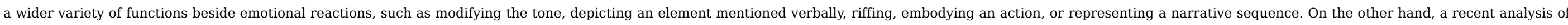

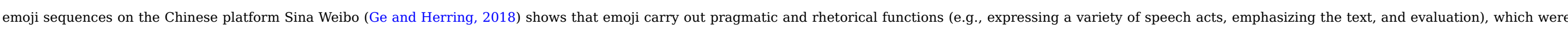

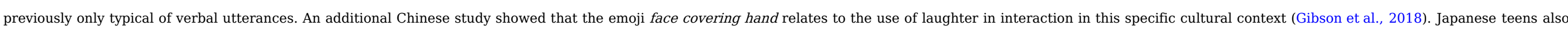
consider relational concerns in the use of emoji to be key, as they carefully use these pictographs to manage the communication climate and to convey a proper image of themselves (Sugiyama, 2015).

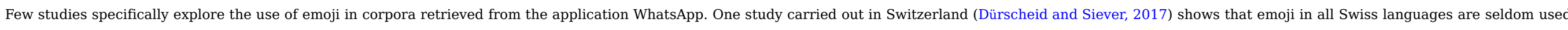

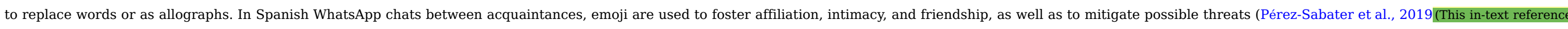

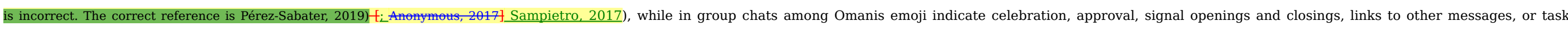
completion (Al Rashdi, 2018).

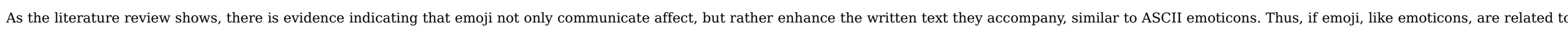

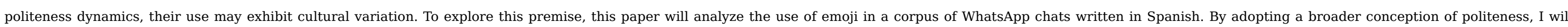

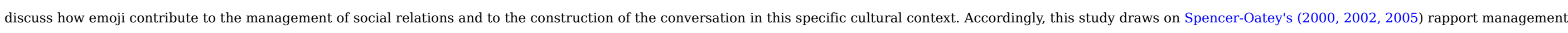
framework, which will be described in the following section.

\section{Rapport management}

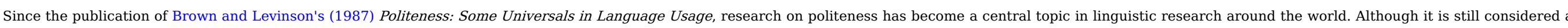

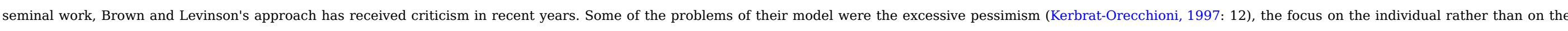

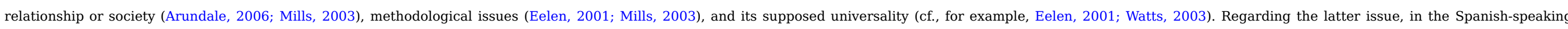

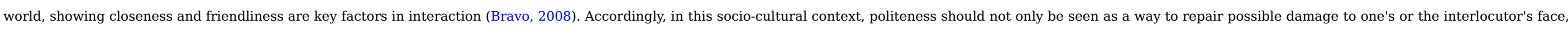
but also as a way to enhance social relations, especially with family or friends (Hernández-Flores, 1999).

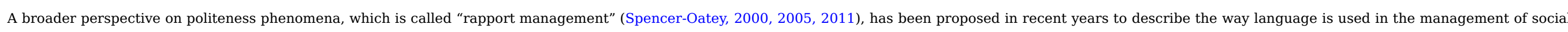

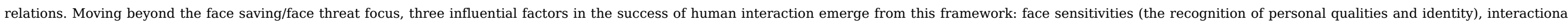

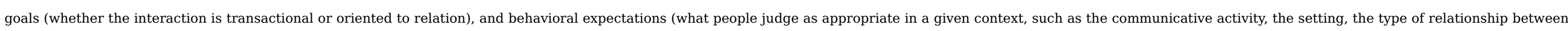




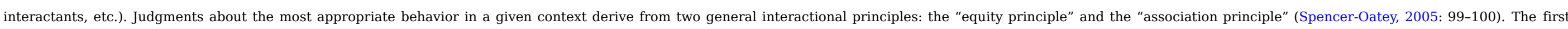

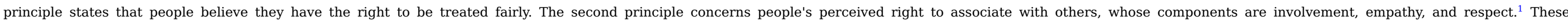

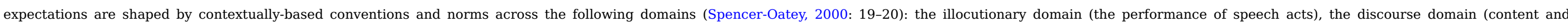

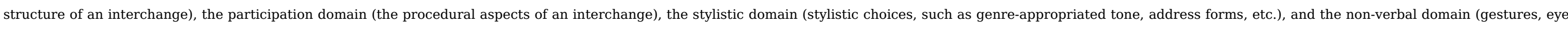
contact, proxemics, etc.).

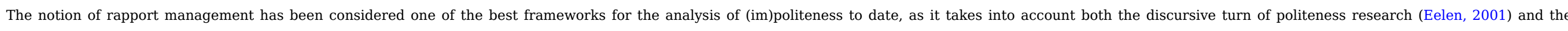

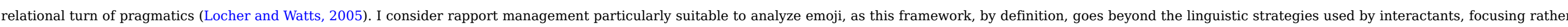

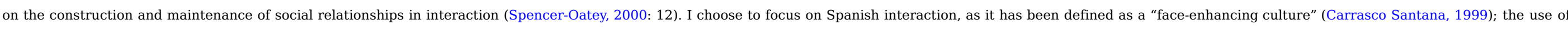

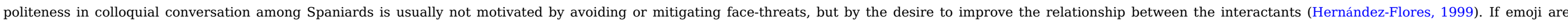

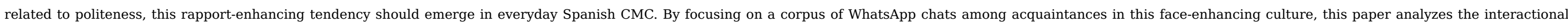
work done by participants using emoji, moving beyond the links between emoji and emotions, and emoji and speech acts (see Gibson et al., 2018 for similar criticism of preceding emoji research).

\section{Methodology}

\subsection{Data and data collection}

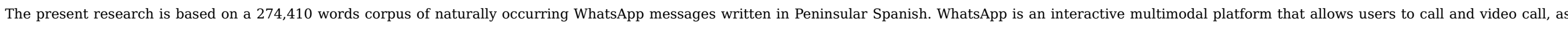

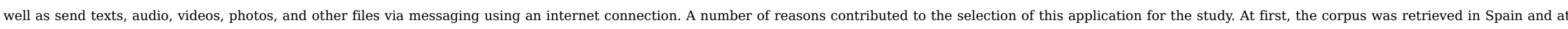

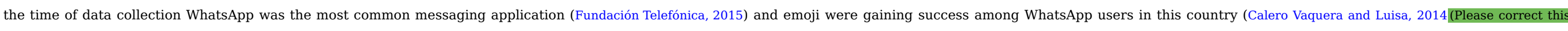

\section{reference: (Calero Vaquera, 2014))).}

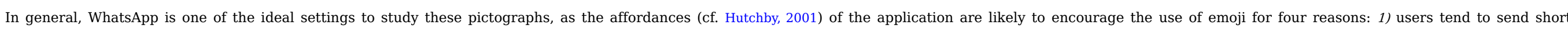

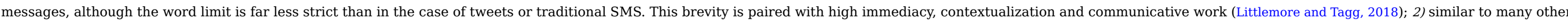

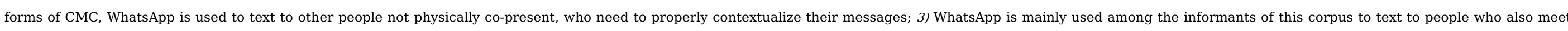

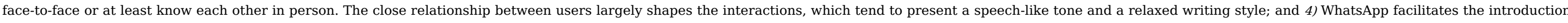
of emoji, as a wide range of pictographs is available and the list of emoji is easily accessible from the keyboard.

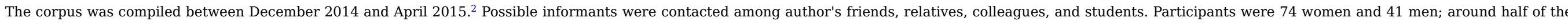

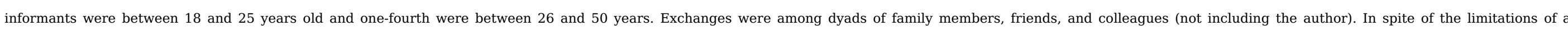

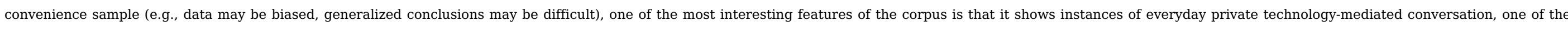

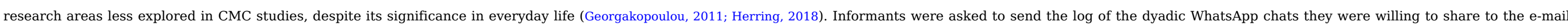
address of the author, giving their informed consent to participate in the research at the same time. Group chats were excluded, as it was not possible to obtain informed consent from all the users.

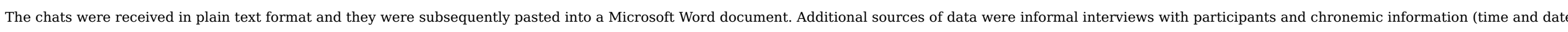

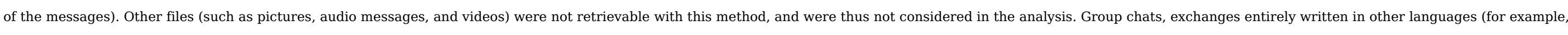
in Catalan), or incomplete conversations (e.g. screenshots) were excluded.

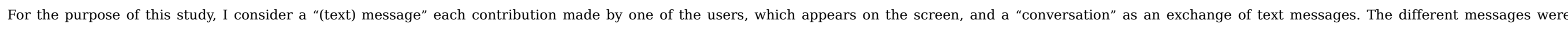

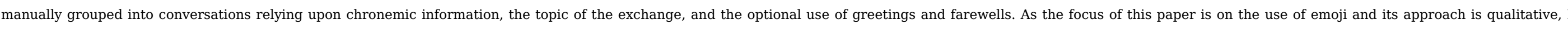

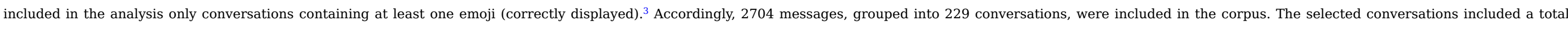

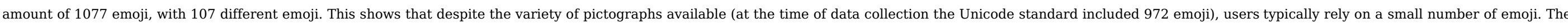

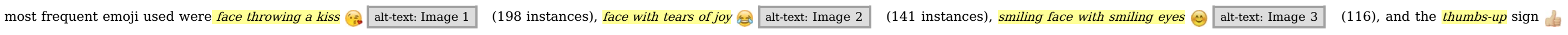




\subsection{Procedure}

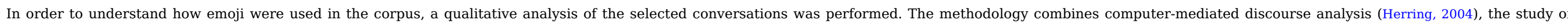

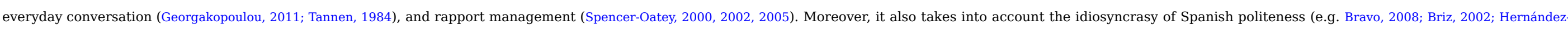

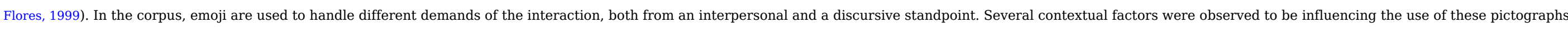

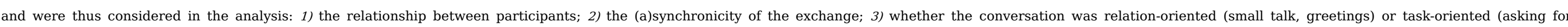

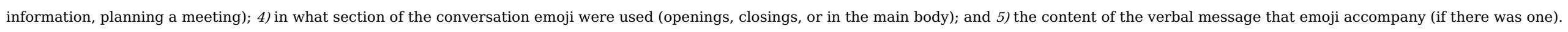

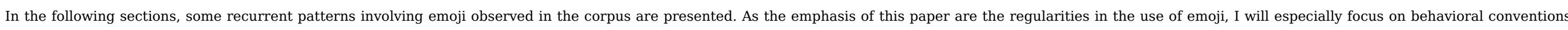

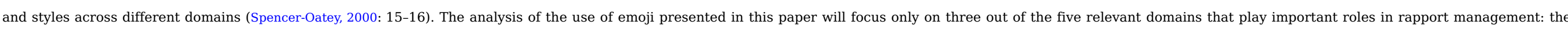

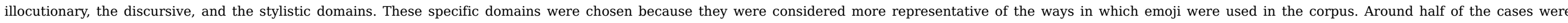

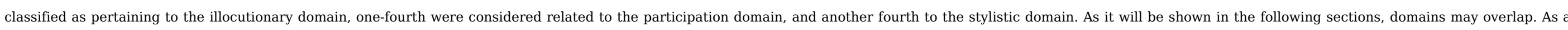
consequence, absolute or relative frequencies are not presented: the analysis exposes trends in the use of emoji and not strict categorizations of the corpus.

\section{Discussion of results}

\subsection{Illocutionary domain}

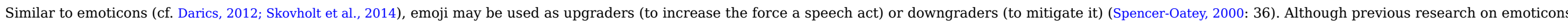

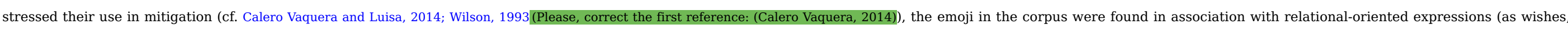

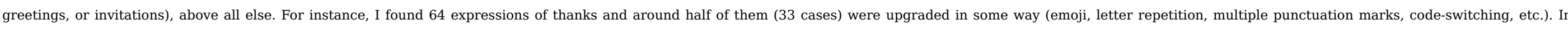

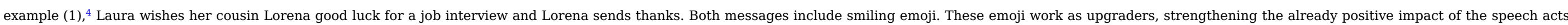
of wishing and thanking, while fostering the affiliation between the interlocutors.

(1)

\begin{tabular}{|c|c|c|c|c|}
\hline 1. & 17.12 .2014 & $8: 07$ & Laura: & $\begin{array}{l}\text { Es hoy la entrevista? Muuuucha suerte!! (4) alt-text: Image } \\
3\end{array}$ \\
\hline & & & & Is the interview today? Gooood luck!! \\
\hline \multirow[t]{2}{*}{2.} & 17.12 .2014 & $9: 22$ & Lorena: & 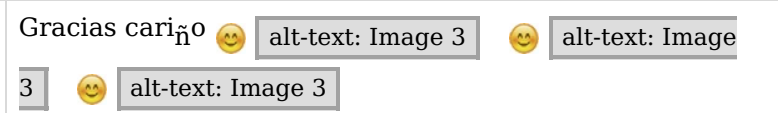 \\
\hline & & & & $\begin{array}{l}\text { Thanks honey () alt-text: Image } 3 \text { alt-text: Image } 3 \\
\text { (ㅅ) alt-text: Image } 3\end{array}$ \\
\hline
\end{tabular}

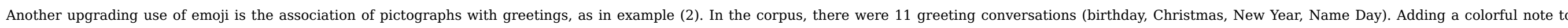

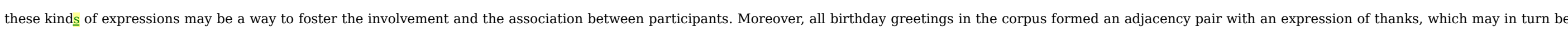

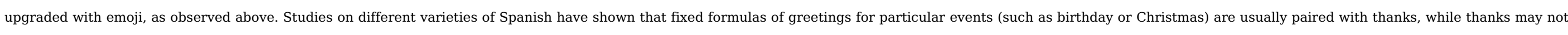
follow other kinds of interactional well-wishing, without being considered rude (Dimitrescu, 2004). 


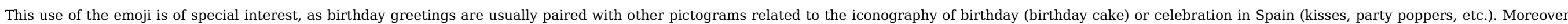

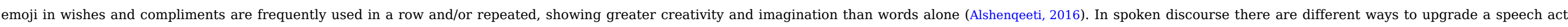

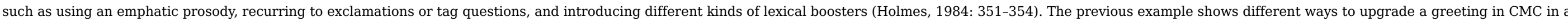

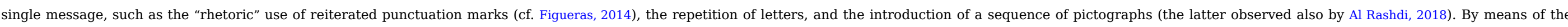

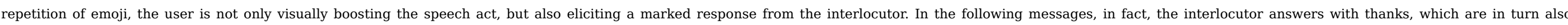

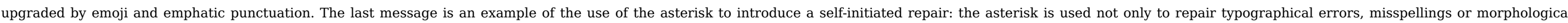
mistakes in CMC (Collister, 2011: 919), but also an inaccurate selection of emoji.

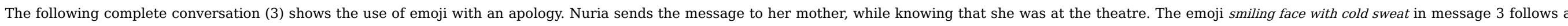
verbal apology, as perdón is a typical illocutionary force indicating device (Blum-Kulka, 1989) for apology in Spanish

(3)

\begin{tabular}{|c|c|c|c|c|}
\hline 1. & 08.11 .2014 & 20:06 & Nuria: & M lo he desinstalado \\
\hline \multirow[t]{3}{*}{2.} & 08.11 .2014 & 20:06 & Nuria: & I've uninstalled it \\
\hline & & & & No mola \\
\hline & & & & Don't like it \\
\hline \multirow[t]{2}{*}{3.} & 08.11 .2014 & 20:06 & Nuria: & Ay perdon q estais en la obra! 상 alt-text: Image 6 \\
\hline & & & & Oh sorry, u're at the show! 사 alt-text: Image 6 \\
\hline
\end{tabular}

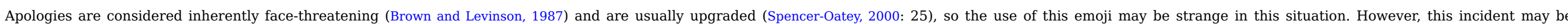

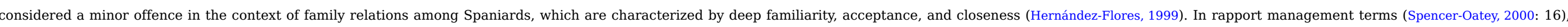

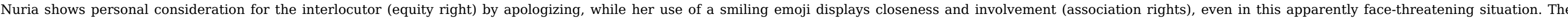

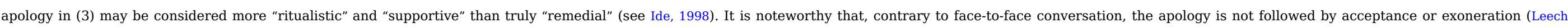
2014: 130): silence terminates the incident.

\subsection{Discourse domain}

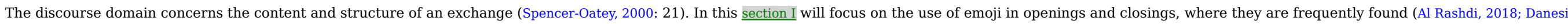

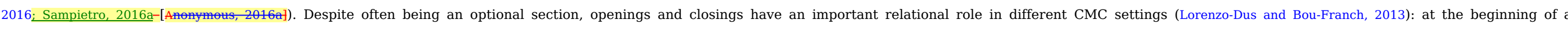

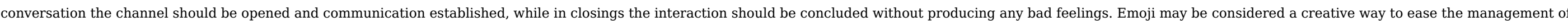




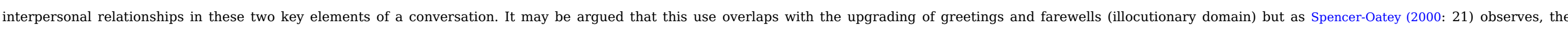
different domains of rapport management are often interrelated.

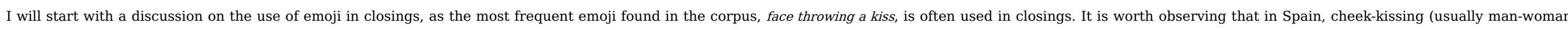

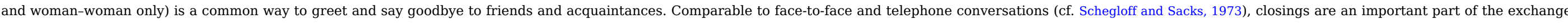

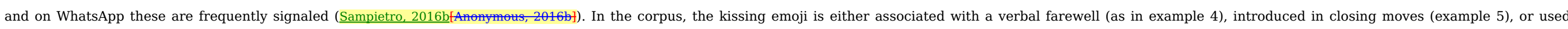
without any other verbal content to close the conversation (as in the last message of example 5).

(4)

\begin{tabular}{|c|c|c|c|c|}
\hline \multirow[t]{2}{*}{1.} & 05.01 .15 & $08: 27$ & Carmen: & Mónica! Buenos días! \\
\hline & & & & Mónica! Good morning! \\
\hline \multirow[t]{2}{*}{2.} & 05.01 .15 & $08: 27$ & Carmen: & A lo largo de la mañana de hoy bajaré al gym \\
\hline & & & & I'm going to the gym throughout this morning \\
\hline \multirow[t]{2}{*}{3.} & 05.01 .15 & $08: 28$ & Carmen: & Lo digo por si quieres bajar \\
\hline & & & & I'll tell you in case you want to go as well \\
\hline \multirow[t]{2}{*}{4.} & 05.01 .15 & $08: 28$ & Carmen: & Besos 2 alt-text: Image 1 \\
\hline & & & & Kisses alt-text: Image 1 \\
\hline \multirow[t]{2}{*}{5.} & 05.01 .15 & $08: 28$ & Carmen: & Ya me dices! \\
\hline & & & & You tell me! \\
\hline \multirow[t]{2}{*}{6.} & 05.01 .15 & $12: 21$ & Mónica: & Me imagino que ya habras bajado no ?? \\
\hline & & & & I guess you've already gone, didn't you?? \\
\hline \multirow[t]{2}{*}{7.} & 05.02 .15 & $12: 21$ & Mónica & Esq me he levantado hoy un poco tarde \\
\hline & & & & The thing is, I woke up a little late today $[\ldots]$ \\
\hline
\end{tabular}

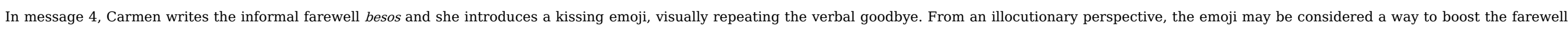

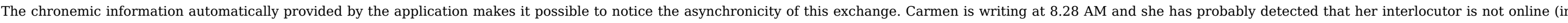

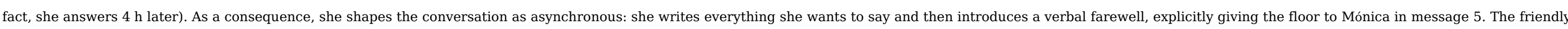

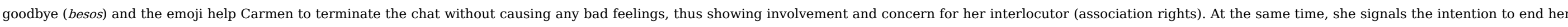

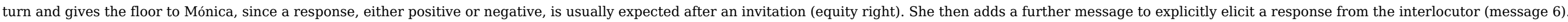

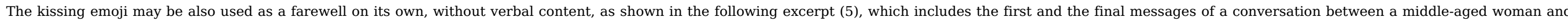
her sister-in-law. In this case, the chronemic information shows that the conversation is synchronous, as the 18 messages of this conversation were sent in less than 5 min.

(5)

\begin{tabular}{|c|c|c|c|c|}
\hline 1. & 18.03.15 & 19:09 & Marta: & $\begin{array}{l}\text { Salis esta noche? } \\
\text { Are you hanging out tonight? [...] }\end{array}$ \\
\hline 14. & 18.03 .15 & 19:14 & Inés: & Si queréis quedamos para para el finde y cenamos \\
\hline & & & & $\begin{array}{l}\text { If you want, we can meet next weekend and have dinner } \\
\text { together }\end{array}$ \\
\hline
\end{tabular}




\begin{tabular}{|c|c|c|c|c|}
\hline 15. & 18.03.15 & 19:14 & Marta: & Vale ya lo hablamos el viernes si quereís \\
\hline & & & & Okay, we'll talk about it on Friday if you want \\
\hline 16. & 18.03 .15 & 19:14 & Inés: & Ok \\
\hline \multirow[t]{2}{*}{17.} & 18.03 .15 & 19:14 & Inés: & Hablamos alt-text: Image 1 \\
\hline & & & & Let's talk :: alt-text: Image 1 \\
\hline 18. & 18.03.15 & 19:14 & Marta: & $\begin{array}{l}\text { alt-text: Image } 4 \\
\text { Image } 1\end{array}$ \\
\hline
\end{tabular}

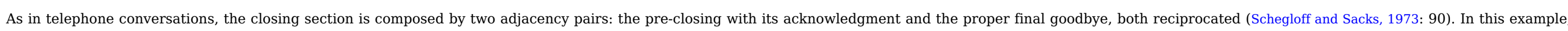

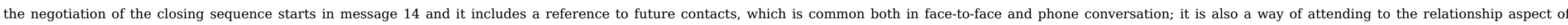

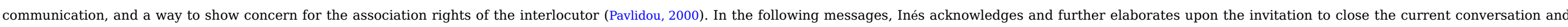

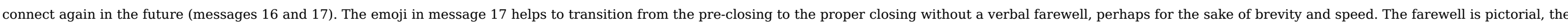

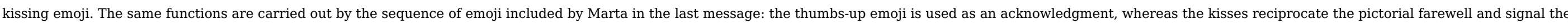
end of the conversation.

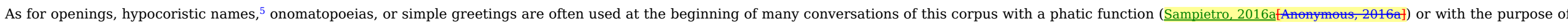

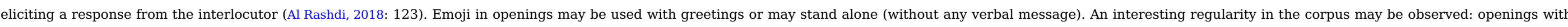
standalone emoji usually start an informal social-oriented exchange between participants, as in the following excerpt (6).

(6)

\begin{tabular}{|c|c|c|c|c|}
\hline \multirow[t]{2}{*}{1.} & \multirow[t]{2}{*}{19.02 .15} & \multirow[t]{2}{*}{ 21:23 } & \multirow[t]{2}{*}{ Domínguez: } & 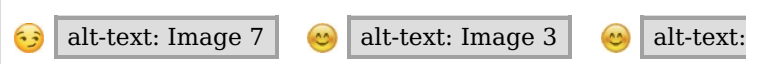 \\
\hline & & & & Image 3 \\
\hline \multirow[t]{2}{*}{2.} & 19.02 .15 & $22: 29$ & Manuela: & Eyyy 3 alt-text: Image $7 \bigcirc$ alt-text: Image 7 \\
\hline & & & & \begin{tabular}{llll} 
Неууу & alt-text: Image 7 & alt-text: Image 7 \\
\cline { 2 - 3 }
\end{tabular} \\
\hline \multirow[t]{3}{*}{3 . } & 19.02 .15 & $22: 29$ & Domínguez: & $\begin{array}{l}\text { Como esta mi valencianita favorita } \\
\begin{array}{l}\text { alt-text: Image } 7 \\
\text { alt-text: Image } 8\end{array}\end{array}$ \\
\hline & & & & \multirow{2}{*}{$\begin{array}{l}\text { How's my favorite Valencian girl } 3 \text { alt-text: Image } 7 \\
\begin{array}{|l|l|}\text { alt-text: Image } 7 & \text { alt-text: Image } 8\end{array}\end{array}$} \\
\hline & & & & \\
\hline \multirow[t]{2}{*}{4.} & 19.02 .15 & $22: 30$ & Manuela: & Bieeeen y voss?? alt-text: Image 3 \\
\hline & & & & Fiiiine and you?? (-) alt-text: Image 3 \\
\hline \multirow[t]{3}{*}{5.} & \multirow[t]{3}{*}{19.02 .15} & \multirow[t]{3}{*}{ 22:30 } & \multirow[t]{3}{*}{ Domínguez: } & $\begin{array}{lll}\text { Molto bene } & \text { alt-text: Image } 9 \\
\end{array}$ \\
\hline & & & & (4) alt-text: Image 3 , ahora mismo fallitas 4 alt-text: \\
\hline & & & & \begin{tabular}{|l|} 
Image 9 \\
\end{tabular} \\
\hline
\end{tabular}




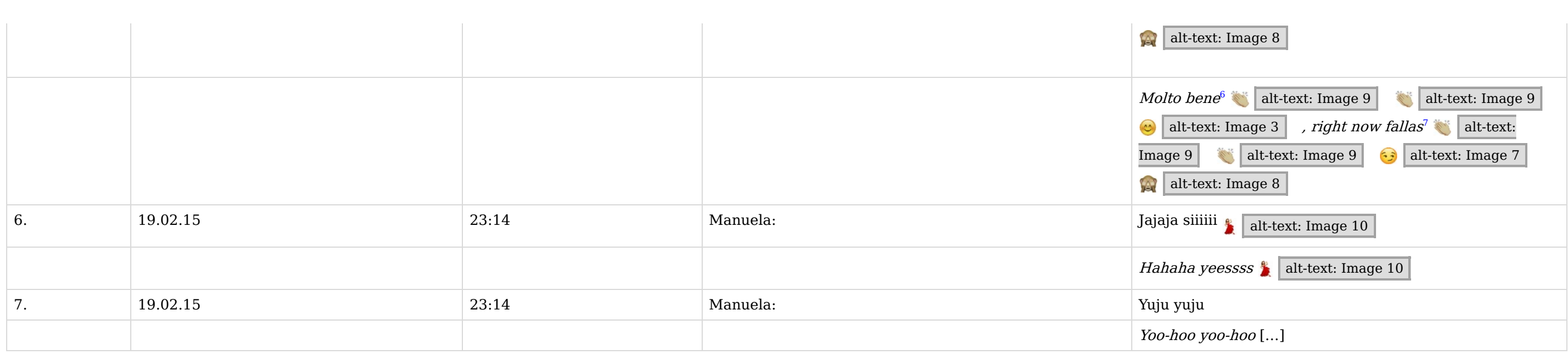

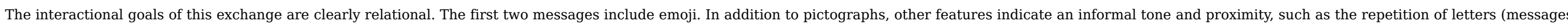

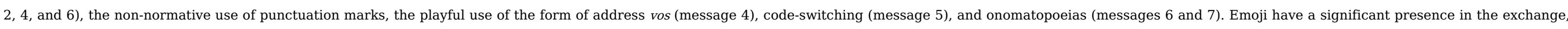

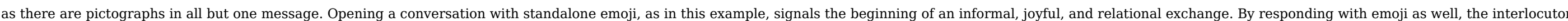
accepts the negotiation of the sequence of phatic communion (Coupland et al., 1992) and acknowledges the playful tone.

\subsection{Stylistic domain}

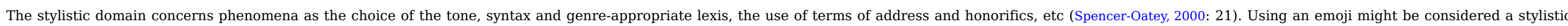

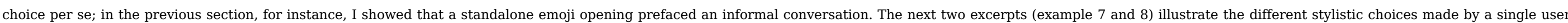
(Esteban) in two different situations, when texting to his boss (7) and to a colleague (8)

(7)

\begin{tabular}{|c|c|c|c|c|}
\hline \multirow[t]{2}{*}{1.} & 03.02 .2014 & $7: 46$ & Esteban: & $\begin{array}{l}\text { Hola } \\
\text { Ayer empecé con fiebre y dolor corporal y esta mañana sigo } \\
\text { igual. } \\
\text { Hoy no iré al trabajo. Intentaré coger hora para el médico. }\end{array}$ \\
\hline & & & & $\begin{array}{l}\text { Hello } \\
\text { Yesterday I started with a fever and body aches and today it's } \\
\text { the same. } \\
\text { I won't be coming in to work today. I'll try to get an } \\
\text { appointment with my doctor. }\end{array}$ \\
\hline \multirow[t]{2}{*}{2.} & 03.02 .2014 & 8:08 & Jaime: & Ok ....avisa a RRHH ... no problem \\
\hline & & & & Ok ....inform HR ... no problem \\
\hline \multirow[t]{2}{*}{3.} & 03.02 .2014 & $8: 24$ & Esteban: & Me puedes pasar el correo? \\
\hline & & & & Could you give me the e-mail? \\
\hline 4. & 03.02 .2014 & $8: 48$ & Jaime: & [e-mail] \\
\hline 5. & 03.02 .2014 & $8: 49$ & Esteban: & Ok \\
\hline 6. & 03.02 .2014 & $8: 50$ & Jaime: & A mejorarse $\ldots . . \cdot$ alt-text: Image 4 \\
\hline
\end{tabular}




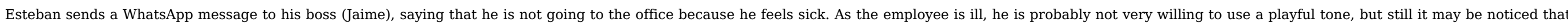

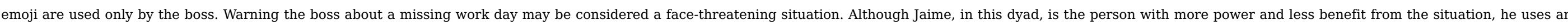

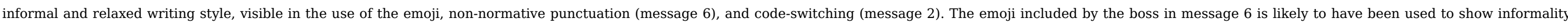

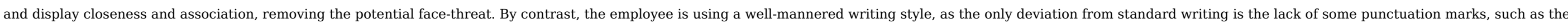

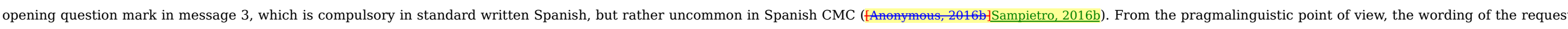

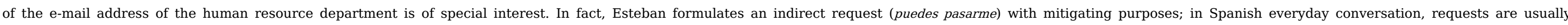

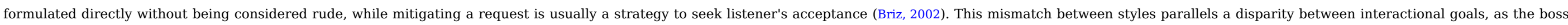
wants to show closeness, understanding, and create an informal relationship with the employees, while Esteban seems to orient the conversation as merely a transactional exchange.

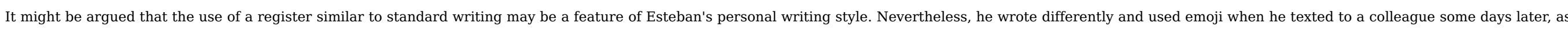
shown in example (8).

(8)

\begin{tabular}{|c|c|c|c|c|}
\hline \multirow[t]{3}{*}{1.} & \multirow[t]{3}{*}{08.02 .15} & \multirow[t]{3}{*}{$13: 57$} & \multirow[t]{3}{*}{ Esteban: } & 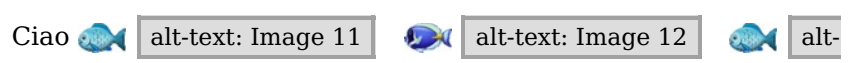 \\
\hline & & & & \multirow{2}{*}{$\begin{array}{l}\text { text: Image } 11 \\
\text { En el ordenador viejo tengo windows vista y una actualización } \\
\text { importante (service pack 1). } \\
\text { ¿La instalo o la evito? }\end{array}$} \\
\hline & & & & \\
\hline & & & & 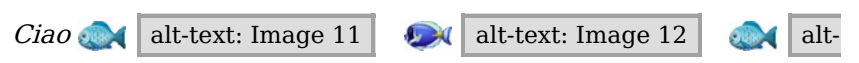 \\
\hline & & & & $\begin{array}{lll}\text { text: Image } 11 & \text { alt-text: Image } 13 \\
\end{array}$ \\
\hline & & & & $\begin{array}{l}\text { In the old computer I have windows vista and an important } \\
\text { update (service pack 1). } \\
\text { Do I install or avoid it? }\end{array}$ \\
\hline 2. & 08.02 .15 & 14:00 & Julián: & Ciao! \\
\hline 3. & 08.02 .15 & 14:00 & Julián: & Es original? \\
\hline & & & & Is it original? \\
\hline 4. & 08.02 .15 & 14:00 & Julián: & Hostia, no entend ${ }_{1}$ a lo de los peces \\
\hline & & & & $\begin{array}{l}\text { Damn, I didn't understand the whole fish thing } \Leftrightarrow \text { alt-text: Image } \\
2\end{array}$ \\
\hline 5. & 08.02 .15 & $14: 00$ & Julián: & No le ira mal $\Leftrightarrow$ alt-text: Image 14 \\
\hline & & & & It won't do wrong $\Leftrightarrow$ alt-text: Image 14 \\
\hline 6. & 08.02 .15 & $14: 00$ & Esteban: & Ok 15 \\
\hline
\end{tabular}




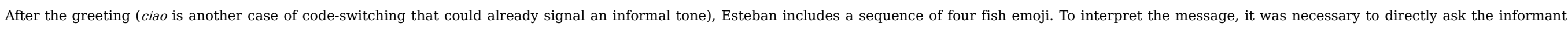

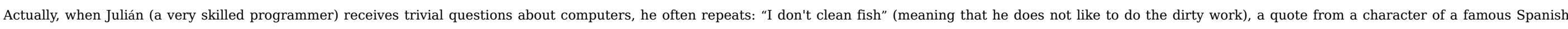

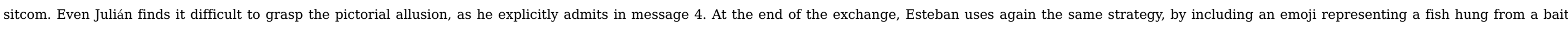
(message 6). Even the last onomatopoeia arrrrrr is a further allusion, as it is a sound repeated by a character of The Simpsons, an animated series admired by both interlocutors.

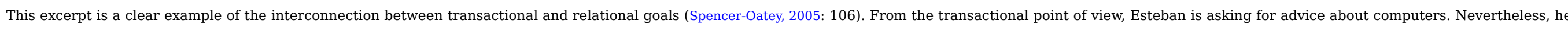

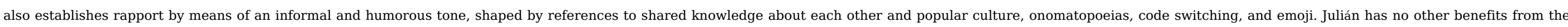

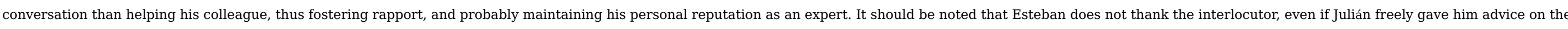

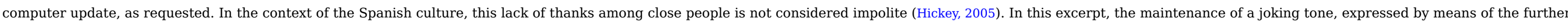

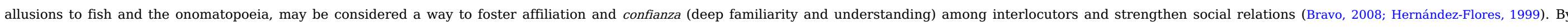

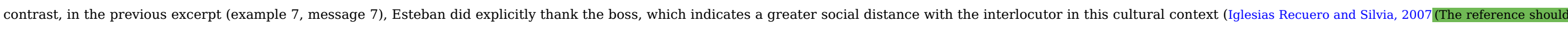
be (Iglesias Recuero, 2007: 26).): 26).

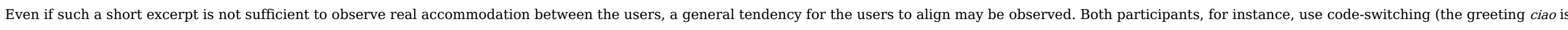

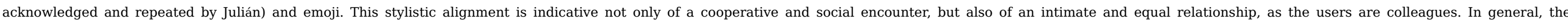
conversation seems to be oriented toward the maintenance of an already harmonious rapport.

\section{Summary and conclusion}

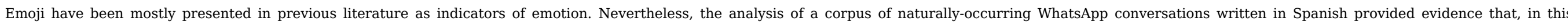

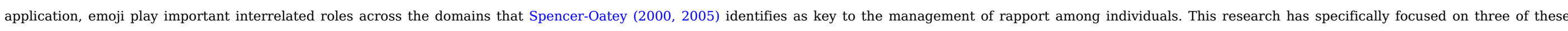
domains: the illocutionary, the discursive, and stylistic domains. To summarize and discuss the findings, the strategies identified throughout the analysis of the corpus are grouped in the following categories:

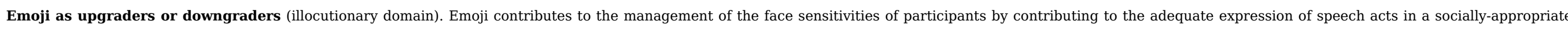
way (Spencer-Oatey, 2000: 23-25)

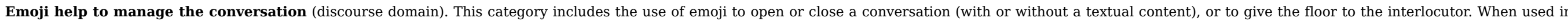

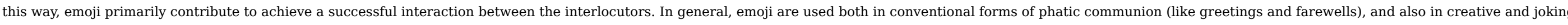

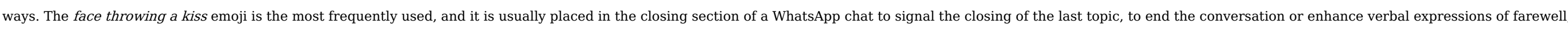

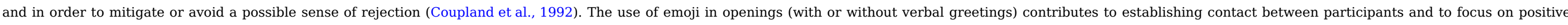
relational goals (Pavlidou, 2000:123). A relevant feature of emoji-only openings in the corpus is that they introduce a socially-oriented exchange.

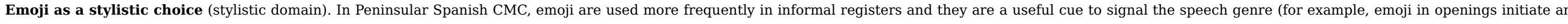

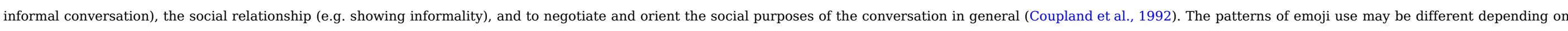
the interlocutor, topic, and technical features as well as concrete circumstances of the exchange.

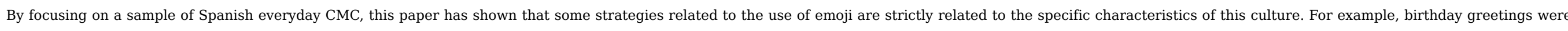

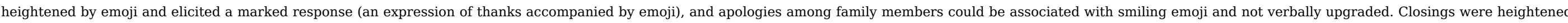

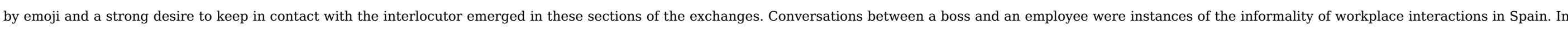

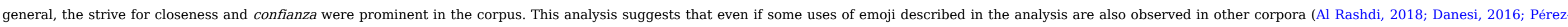




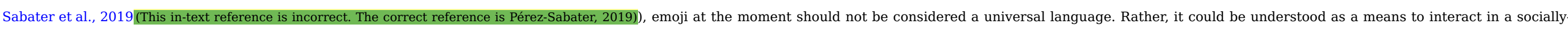
appropriate way, in a given culture.

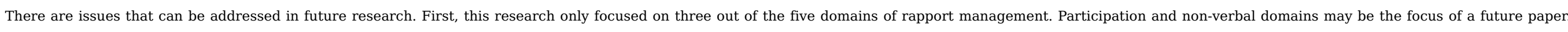

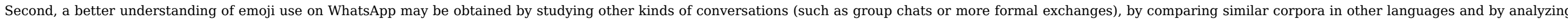

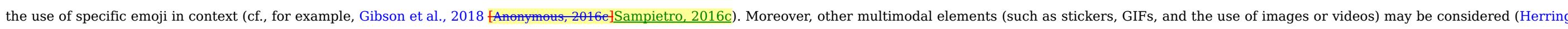

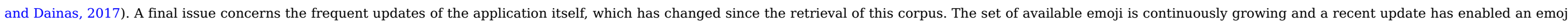
prediction feature, which can profoundly influence the use of emoji in this application. Thus, the results presented in this work should be frequently updated.

\section{Declarations of interest}

None.

\section{Funding}

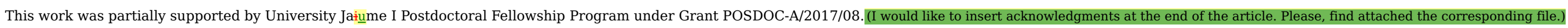

\section{Appendix A. Supplementary data}

Supplementary data to this article can be found online at https://doi.org/10.1016/j.pragma.2019.02.009.

\section{References}

Al Rashdi Fathiya, Functions of emojis in WhatsApp interaction among Omanis. Discourse, Context Media 26, 2018, 117-126 https://doi.org/10.1016/j.dcm.2018.07.001.

Alshenqeeti Hamza, Are emojis creating a new or old visual language for new generations? A socio-semiotic study, Adv. Lang. Lit. Stud. 7, 2016, 56-69 https://doi.org/10.7575/aiac.alls.v.7n.6p.56.

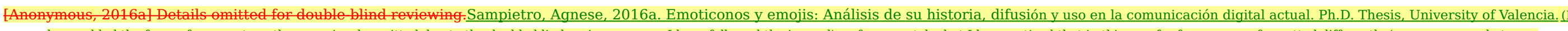

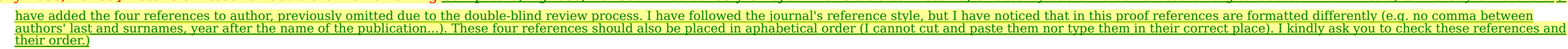

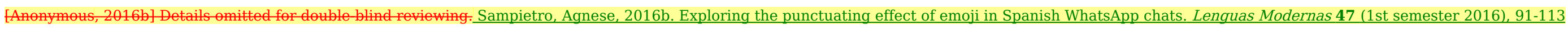
https://lenguasmodernas.uchile.cl/index.php/LM/article/view/45186/47242

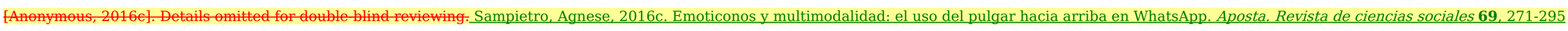
http://www.apostadigital.com/revistav3/hemeroteca/asampietro.pdf

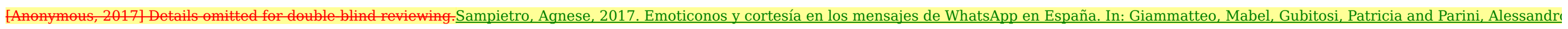
(Eds.), El español en la red. Usos y géneros de la comunicación mediada por computadora. Iberoamericana, Vervuert Verlagsgesellschaft, Madrid, Frankfurt, pp. 279-302.

Arundale Robert B., Face as relational and interactional: a communication framework for research on face, facework, and politeness, J. Politeness Res. Lang. Behav. Cult. 2 (2), 2006, 193-216

https://doi.org/10.1515/PR.2006.011.

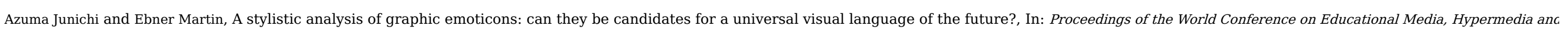
Telecommunications (ED-Media), 2008, Association for the Advancement of Computing in Education (AACE); Vienna, 972-977, 30 June - 4 July 2008.

Blagdon Jeff, How emoji conquered the world. The Verge, 4 March 2013, 2013 http://www.theverge.com/2013/3/4/3966140/how-emoji-conquered-the-world.

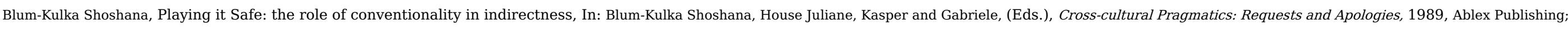
Norwood, 37-70. 
Bravo Diana, The implications of studying politeness in Spanish-speaking contexts: A discussion, Pragmatics 18, 2008, 577-603 https://doi.org/582-925-1-PB.

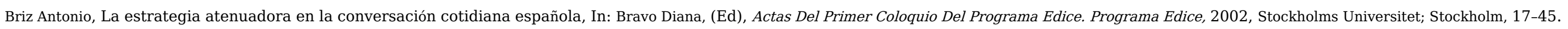

Brown Penelope and Levinson Stephen C., Politeness: Some universals in language usage, 1987, Cambridge University Press; Cambridge.

Calero Vaquera-and Luisa María, El discurso del WhatsApp: entre el Messenger y el SMS, Oralia 17, 2014, 85-114.

Carrasco Santana Antonio, Revisión y evaluación del modelo de cortesía de Brown y Levinson, Pragmalinguistica 7, 1999, 1-44 https://doi.org/10.25267/Pragmalinguistica.1999.i7.01.

Coupland Justine, Coupland Nikolas and Robinson Jeffrey D., "How are you?”: Negotiating phatic communion, Lang. Soc. 21, 1992, 207-230 https://doi.org/10.1017/S0047404500015268.

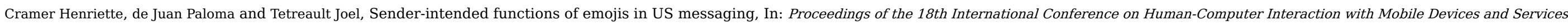
MobileHCI '16, 2016, ACM Press; New York, 504-509 https://doi.org/10.1145/2935334.2935370.

Crystal David, The Penguin Dictionary of Language, second ed., 1999, Penguin; London.

Danesi Marcel, The Semiotics of Emoji: The Rise of Visual Language in the Age of the Internet, 2016, Bloomsbury; London.

Danet Brenda and Herring Susan C., Introduction. Welcome to the multilingual internet, J. Comput. Commun. 9 (1), 2003, 3-39 https://doi.org/10.1111/j.1083-6101.2003.tb00354.x.

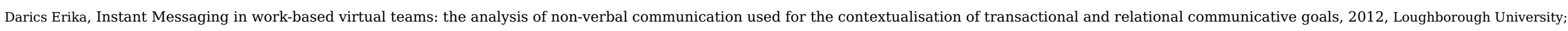
Loughborough.

Derks Daantje, Bos Arjan E.R. and von Grumbkow Jasper, Emoticons and online message interpretation, Soc. Sci. Comput. Rev. 26, 2007, 379-388 https://doi.org/10.1177/0894439307311611.

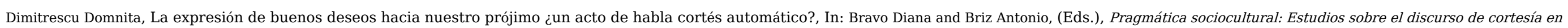
español, 2004, Ariel; Barcelona, 265-284.

Dresner Eli and Herring Susan C., Functions of the nonverbal in CMC: emoticons and Illocutionary force, Commun. Theor. 20, 2010, 249-268 https://doi.org/10.1111/j.1468-2885.2010.01362.x.

Dürscheid Christa and Siever Cristina M., Jenseits des Alphabets - Kommunikation mit Emojis, Z. Ger. Linguist. 45, 2017, 256-285 https://doi.org/10.1515/zgl-2017-0013.

Eelen Gino, A Critique of Politeness Theories, 2001, St. Jerome Publishing; Manchester.

Figueras Carolina, Pragmática de la puntuación y nuevas tecnologías, Normas 4, 2014, 135-160 https://ojs.uv.es/index.php/normas/article/view/4691/4550.

Ge Jing and Herring Susan C., Communicative functions of emoji sequences on Sina Weibo, Clin. Hemorheol. and Microcirc. 23 (11), 2018 https://doi.org/10.5210/fm.v23i11.9413.

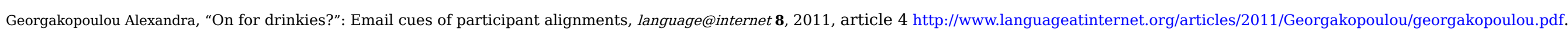

Gibson Will, Huang Pingping and Yu Qianyun, Emoji and communicative action: the semiotics, sequence and gestural actions of 'face covering hand.' discourse, Context Media 26, 2018, 91-99

https://doi.org/10.1016/j.dcm.2018.05.005.

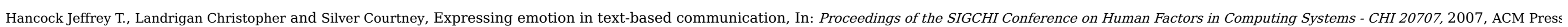
New York, 929-932 https://doi.org/10.1145/1240624.1240764.

Hernández-Flores Nieves, Politeness ideology in Spanish colloquial conversation, Pragmatics 9, 1999, 37-49 https://doi.org/10.1075/prag.9.1.04her.

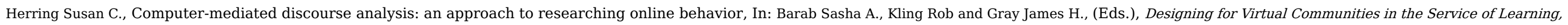
2004, Cambridge University Press; New York, 338-376. 
Herring Susan C., Emergent forms of computer-mediated communication and their global implications, LinguaPax Rev 2017, 2018, 90-130 http://www.linguapax.org/wpcontent/uploads/2018/11/LinguapaxReview2017_web-1.pdf.

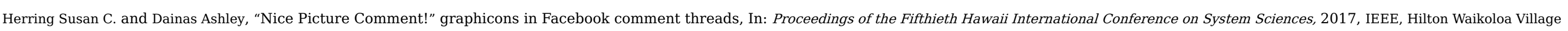
Hawaii, 2185-2194 http://hdl.handle.net/10125/41419.

Hickey Leo, Politeness in Spain: thanks but no 'thanks', In: Hickey Leo and Stewart Miranda, (Eds.), Politeness in Europe, 2005, Multilingual Matters; Clevendon, 317-330.

Hutchby Ian, Technologies, texts and affordances, Sociology 35 (2), 2001, 441-456 https://doi.org/10.1177/S0038038501000219.

Ide Risako, "Sorry for your kindness": Japanese interactional ritual in public discourse, J. Pragmat. 29, 1998, 509-529 https://doi.org/10.1016/S0378-2166(98)80006-4.

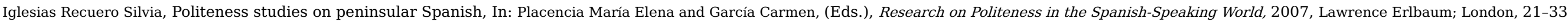

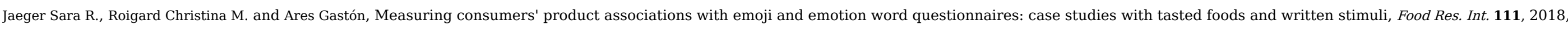
732-747 https://doi.org/10.1016/J.FOODRES.2018.04.010.

Kavanagh Barry, A cross-cultural analysis of Japanese and English non-verbal online communication: the use of emoticons in weblogs, Intercult. Commun. Stud. XIX (3), 2010, 65-80

https://web.uri.edu/iaics/files/05barryKavanagh.pdf.

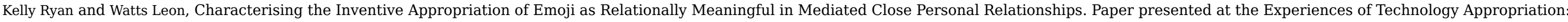
Unanticipated Users, Usage, Circumstances, and Design. Oslo, Norway, 20 September 2015, 2015 https://pdfs.semanticscholar.org/7df9/22c402875f113c6ce1c32c744a59171fb275.pdf? ga=2.183141350.1583998080.1549294982-1164004195.1549294982.

Kerbrat-Orecchioni Christine, A multilevel approach in the study of talk-in-interaction, Pragmatics 7 (1), 1997, 1-20 https://doi.org/10.1075/prag.7.1.01 ker.

Leech Geoffrey, The Pragmatics of Politeness. Oxford Scholarship Online, 2014 https://doi.org/10.1093/acprof:0so/9780195341386.001.0001.

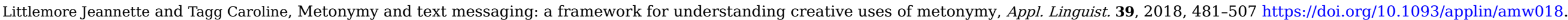

Locher Miriam A. and Watts Richard J., Politeness theory and relational work, J. Politeness Res. Lang. Behav. Cult. 1 (1), 2005, 9-33 https://doi.org/10.1515/jplr.2005.1.1.9.

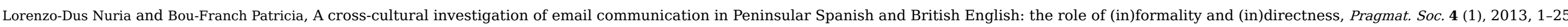
https://doi.org/10.1075/ps.4.1.01lor.

Marengo Davide, Giannotta Fabrizia and Settanni Michele, Assessing personality using emoji: an exploratory study, Pers. Indiv. Differ. 112, 2017, 74-78 https://doi.org/10.1016/J.PAID.2017.02.037.

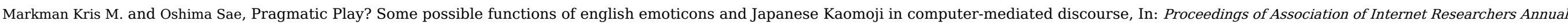
Conference 8.0, 2007, Let's Play; Vancouver, Canada, 1-19 https://osf.io/preprints/socarxiv/qa764/.

Mills Sara, Gender and Politeness, 2003, Cambridge University Press; Cambridge.

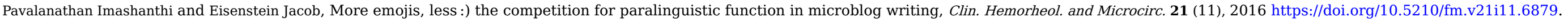

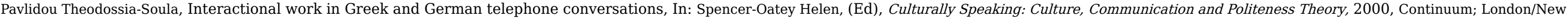
York, 118-135.

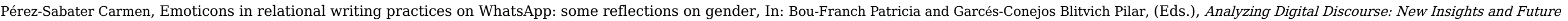
Directions, 2019, Springer International Publishing; Cham, 163-189 https://doi.org/10.1007/978-3-319-92663-6_6. 
Petitjean Cécile and Morel Etienne, “Hahaha”: laughter as a resource to manage WhatsApp conversations, J. Pragmat. 110, 2017, 1-19 https://doi.org/10.1016/j.pragma.2017.01.001.

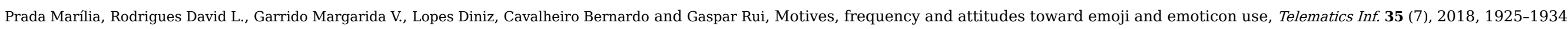
https://doi.org/10.1016/j.tele.2018.06.005.

Ren Wei, Exploring Chinese digital communication, Discourse, Context Media 26, 2018, 1-4 https://doi.org/10.1016/j.dcm.2018.07.002.

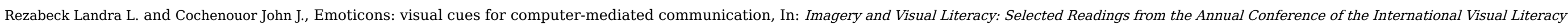

Association, 1995, 371-383, Tempe, Arizona, 26 October 1994 http://files.eric.ed.gov/fulltext/ED380096.pdf.

Riordan Monica A., Emojis as tools for emotion work: communicating affect in text messages, J. Lang. Soc. Psychol. 36 (5), 2017, 549-567 https://doi.org/10.1177/0261927X17704238.

Schegloff Emanuel A. and Sacks Harvey, Opening up closings, Semiotica 8 (4), 1973, 289-327 https://doi.org/10.1515/semi.1973.8.4.289.

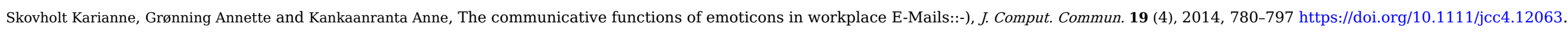

Spencer-Oatey Helen, Culturally speaking: Managing rapport through talk across cultures, 2000, Continuum; London.

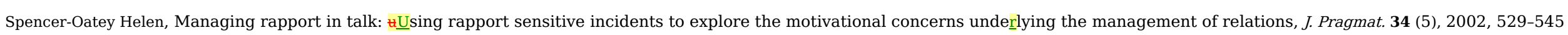

https://doi.org/10.1016/S0378-2166(01)00039-X.

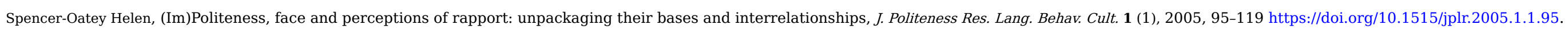

Spencer-Oatey Helen, Conceptualising 'the relational' in pragmatics: insights from metapragmatic emotion and (im)politeness comments, J. Pragmat. 43 (14), 2011, 3565-3578

https://doi.org/10.1016/j.pragma.2011.08.009.

Stark Luke and Crawford Kate, The conservatism of emoji: work, affect, and communication, Soc. Media Soc. 1 (2), 2015, 1-11 https://doi.org/10.1177/2056305115604853.

Sugiyama Satomi, Kawaii meiru and Maroyaka neko: mobile emoji for relationship maintenance and aesthetic expressions among Japanese teens, Clin. Hemorheol. and Microcirc. 20 (10), 2015

https://doi.org/10.5210/fm.v20i10.5826.

Tannen Deborah, Conversational style: Analyzing talk among friends, 1984, Ablex; Norwood.

Telefónica Fundación, La Sociedad de la Información en España 2014, 2015, Ariel; Barcelona.

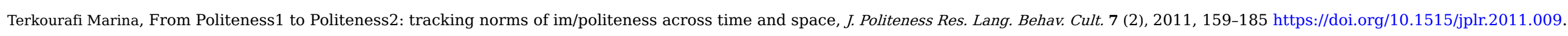

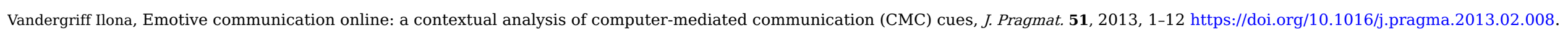

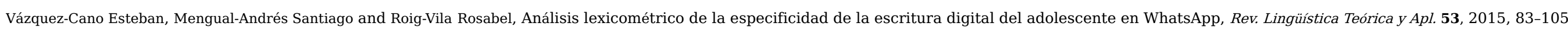

https://doi.org/10.4067/S0718-48832015000100005.

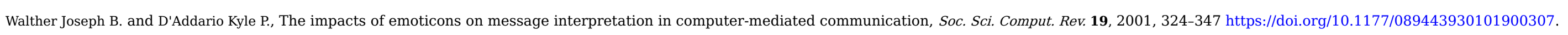

Watts Richard J., Politeness, 2003, Cambridge University Press; Cambridge.

Wilson Andrew, A pragmatic device in electronic communication, J. Pragmat. 19 (4), 1993, 389-398 https://doi.org/10.1016/0378-2166(93)90098-A.

Yus Francisco, Not all emoticons are created equal, Ling. em (Dis)curso 14, 2014, 511-529 https://doi.org/10.1590/1982-4017-140304-0414.

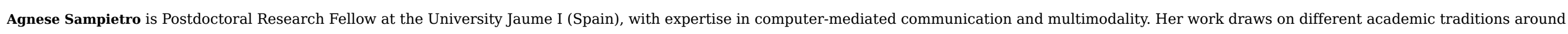

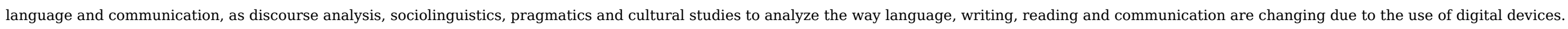




\title{
Footnotes
}

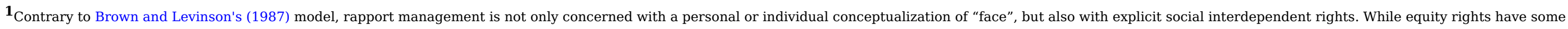

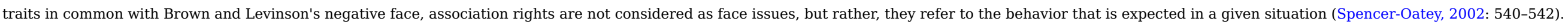

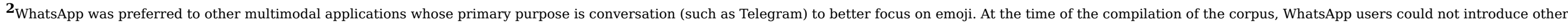

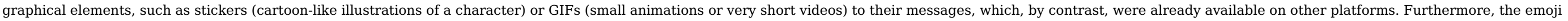
prediction feature (an automatic suggestion of a possible emoji depending on what the user is typing) was not yet available.

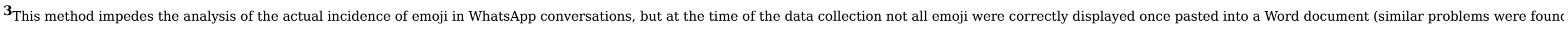
by Petitjean and Morel (2017), and the sample of respondents was not probabilistic. For a stylometric analysis of a large corpus of WhatsApp messages among teens in Spain, cf. Vázquez-Cano et al. (2015).

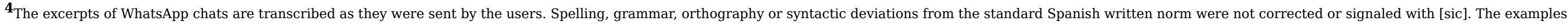
were translated into English by the author trying to mirror the most relevant features of the original in Spanish. For privacy purposes, the names of the participants have been changed.

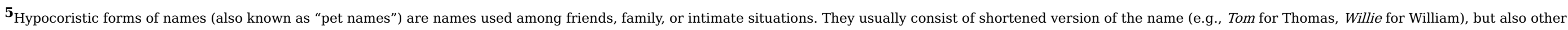
names (e.g. honey) or new creations may be found (Crystal, 1999: 152).

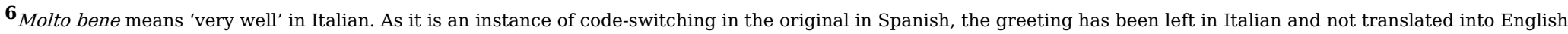

${ }^{7}$ Fallas is a celebration in the city of Valencia (Spain) held every year in March in commemoration of Saint Joseph

\section{Appendix A. Supplementary data}

\author{
The following is the Supplementary data to this article:
}

\author{
Multimedia Component 1
}

Multimedia component 1

alt-text: Multimedia component 1

\section{Highlights}

- Analysis of some of the functions of emoji in WhatsApp chats written in Spanish.

- Emoji upgrade or downgrade different speech acts.

- Emoji help to manage the conversation, e.g. in openings and closings.

- Emoji are also a stylistic choice.

\section{Queries and Answers}

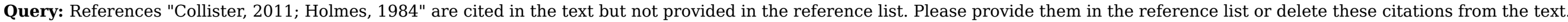
Answer: Here are the references (I cannot edit the reference list) Collister, Lauren B., 2011. *-repair in Online Discourse. Journal of Pragmatics, 43(3), 918-921 https://doi.org/10.1016/j.pragma.2010.09.025

Holmes, Janet, 1984. Modifying illocutionary force. Journal of Pragmatics

8, 3, 345-365 https://doi.org/10.1016/0378-2166(84)90028-6.

Query: Have we correctly interpreted the following funding source(s) you cited in your article: University Jaime I Postdoctoral Fellowship Program? 
Answer: Yes, you correctly interpreted the founding sources, but the name of the institution is University Jaume I (you wrote Jaime I). I edited it.

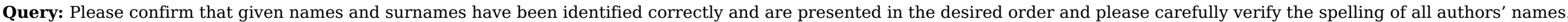
Answer: Yes

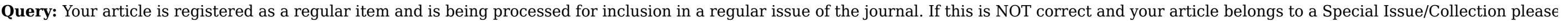
contact b.ganeshan@elsevier.com immediately prior to returning your corrections.

Answer: Yes 